\title{
Identification of Optimal Risky Portfolios for Hedge Fund
}

\author{
*Runsheng Rong ${ }^{1}$, Yongwei Yang ${ }^{2}$, Mengru $\mathrm{He}^{3}$, Dingyin $\mathrm{Hu}^{4}$, Zhenting $\mathrm{Gu}^{5}$ \\ ${ }^{1}$ School of Computer Science and Mathematics, Arcadia University, Glenside, PA 19038, USA \\ ${ }^{2}$ San Domenico School, San Anselmo, CA, 94960, USA, yyang22@sandomenico.org \\ ${ }^{3}$ School of Mathematics and Statistics, Miami University, Oxford, OH, 45056,USA, hem15@miamioh.edu \\ ${ }^{4}$ Tung Wah senior High School, Dongguan, Guangdong, 523000, China, 2197068459@qq.com \\ ${ }^{5}$ Zibo Experimental High School International Department, Zibo, Shandong, 255000, China, 2958868741@qq.com \\ *hatsunemiku9630@gmail.com
}

\begin{abstract}
The hedge fund market, stock market and bond market all have high risks. The purpose of our project is to build the optimal risky portfolio consisting of ten different strategies (which includes Arbitrage, CTA/Managed Futures etc.) to diversify risk and gain a high return, and we also conducted a 5-fold cross-validation test to validate if our method works accurately for all the periods. Using more strategies can help us carry out comprehensive analysis on different situations, periods and types of investment portfolio, so as to disperse risks and obtain high returns, also, ensure the diversity of portfolio and a lower risk.
\end{abstract}

Keywords: hedge fund, strategy, optimal risky portfolio, 5-fold cross-validation

\section{INTRODUCTION}

Currently, people are more interested in investing in hedge funds, since hedge funds' returns are very different from those of standard asset classes, which makes them attractive to investors looking for a diversified and balanced portfolio [1-2]. Therefore, the research on hedge fund investment naturally focuses on finding the optimal proportion of hedge fund investment, measuring the performance of hedge fund, identifying the risk factors of hedge fund, and finally constructing the optimal hedge fund investment portfolio [1-6].

This research adopted ten different strategies to set up the optimal risky portfolio and we analyzed monthly return data and tested those with a 5-fold cross-validation test. Cross-validation is a statistical method used to validate statistical models. It is commonly used in applied machine learning to compare and select a model for a given predictive modeling problem because it is easy to understand, easy to implement, and results in skill estimates that generally have a lower bias than other methods. So it's a way to tell whether the method of selecting the optimal risky portfolio works [7].

\section{METHODOLOGY}

Firstly, we downloaded the original monthly return data of nine different strategies of hedge funds for the period between January 2,000 to April 2,021 from EurekaHedge and the adjusted closing price data of S\&P 500 for the same period from Yahoo Finance, and we calculated the original monthly return rates of S\&P 500 for each month by dividing each price by the price for the previous month. Secondly, we subtracted the risk-free rates (from Fama-French Table) for each month from the original monthly return rates to get the monthly excess return rates, and made a new sheet containing these numbers. Then we calculated the monthly average excess return rates and monthly standard deviation of strategies selected by us. The next step was to deduce the average monthly excess return rates from the original monthly return rates data to create a new spreadsheet. Then we created a spreadsheet containing the covariance matrix, we also made the correlation coefficient matrix. Nextly, we added a number (depending on the maximum value in the diagonal) to every value in the diagonal of the covariance matrix to create the adjusted covariance matrix according to robust optimization theorem and then we calculated the inverse covariance matrix. Finally, we created a new vector, and we divided each value of the 
sum of all the values in the vector to calculate the optimal risky portfolio, we also got the expected monthly return rate for this portfolio, and we calculated the monthly standard deviation. We multiplied the monthly return by 12 to get the expected annualized return of the optimal risky portfolio, and multiplied the monthly standard deviation by the square root of 12 to get the annualized standard deviation, then we also calculated the annualized Sharpe ratio by dividing the expected annualized return rate by the annualized standard deviation.

We also did a 5-fold cross-validation test on our optimal portfolio. We allocated 256 monthly return datas into 5 sets of the same amount of datas, then we firstly set the first set which contained data from January 2,000 to April 2,004 as test set, and set other sets as training set, then we ran the procedure for building the optimal risky portfolio on the training set, and used this portfolio on the test set to calculate the excess return and standard deviation, we repeated such step for 5 times by setting each of the 5 sets as test set for once and only once.

\section{DATA}

The research data is shown in Table 1-4. In Table 1, we presented the average monthly excess return rates for our ten strategies. Table 2 shows the monthly standard deviation rates of those strategies. As shown in Table 3, the chosen strategies' annualized Sharpe ratio are presented. Table 4 shows the correlation coefficient matrix consisting of our selected strategies.

Table 1. shows Average Monthly Excess Return Rate

\begin{tabular}{|c|c|c|c|c|c|c|c|c|c|}
\hline Arbitrage & $\begin{array}{c}\text { CTA/Managed } \\
\text { Futures }\end{array}$ & $\begin{array}{c}\text { Distressed } \\
\text { Debt }\end{array}$ & $\begin{array}{c}\text { Fixed } \\
\text { Income }\end{array}$ & $\begin{array}{c}\text { L/S } \\
\text { Equities }\end{array}$ & Macro & $\begin{array}{c}\text { Multi- } \\
\text { Strategy }\end{array}$ & $\begin{array}{c}\text { Relative } \\
\text { Value }\end{array}$ & $\begin{array}{c}\text { Long- } \\
\text { Only } \\
\text { Absolute } \\
\text { Return } \\
\text { Fund }\end{array}$ & $\begin{array}{c}\text { S\&P } \\
500\end{array}$ \\
\hline $0.20 \%$ & $0.30 \%$ & $0.29 \%$ & $0.24 \%$ & $0.28 \%$ & $0.23 \%$ & $0.24 \%$ & $0.16 \%$ & $0.64 \%$ & $0.40 \%$ \\
\hline
\end{tabular}

Table 2. shows Monthly Standard Deviation

\begin{tabular}{|c|c|c|c|c|c|c|c|c|c|}
\hline Arbitrage & $\begin{array}{c}\text { CTA/Managed } \\
\text { Futures }\end{array}$ & $\begin{array}{c}\text { Distressed } \\
\text { Debt }\end{array}$ & $\begin{array}{c}\text { Fixed } \\
\text { Income }\end{array}$ & $\begin{array}{c}\text { L/S } \\
\text { Equities }\end{array}$ & Macro & $\begin{array}{c}\text { Multi- } \\
\text { Strategy }\end{array}$ & $\begin{array}{c}\text { Relative } \\
\text { Value }\end{array}$ & $\begin{array}{c}\text { Long- } \\
\text { Only } \\
\text { Absolute } \\
\text { Return } \\
\text { Fund }\end{array}$ & $\begin{array}{c}\text { S\&P } \\
500\end{array}$ \\
\hline $0.92 \%$ & $2.20 \%$ & $1.45 \%$ & $1.16 \%$ & $1.99 \%$ & $1.22 \%$ & $1.34 \%$ & $1.32 \%$ & $3.79 \%$ & $4.37 \%$ \\
\hline
\end{tabular}

Table 3. shows Annualized Sharpe Ratio

\begin{tabular}{|c|c|c|c|c|c|c|c|c|c|}
\hline Arbitrage & $\begin{array}{c}\text { CTA/Ma } \\
\text { naged } \\
\text { Futures }\end{array}$ & $\begin{array}{c}\text { Distress } \\
\text { ed Debt }\end{array}$ & $\begin{array}{c}\text { Fixed } \\
\text { Income }\end{array}$ & $\begin{array}{c}\text { L/S } \\
\text { Equities }\end{array}$ & Macro & $\begin{array}{c}\text { Multi- } \\
\text { Strategy }\end{array}$ & $\begin{array}{c}\text { Relative } \\
\text { Value }\end{array}$ & $\begin{array}{c}\text { Long- } \\
\text { Only } \\
\text { Absolute } \\
\text { Return } \\
\text { Fund }\end{array}$ & S\&P 500 \\
\hline 0.74 & 0.47 & 0.69 & 0.71 & 0.49 & 0.66 & 0.62 & 0.42 & 0.59 & 0.32 \\
\hline
\end{tabular}

Table 4. shows Correlation Coefficient

\begin{tabular}{|c|c|c|c|c|c|c|c|c|c|c|}
\hline & $\begin{array}{c}\text { Arbitrag } \\
\mathrm{e}\end{array}$ & $\begin{array}{l}\text { CTA/M } \\
\text { anaged } \\
\text { Futures }\end{array}$ & $\begin{array}{l}\text { Distress } \\
\text { ed Debt }\end{array}$ & $\begin{array}{l}\text { Fixed } \\
\text { Income }\end{array}$ & $\begin{array}{c}\text { L/S } \\
\text { Equities }\end{array}$ & Macro & $\begin{array}{c}\text { Multi- } \\
\text { Strateg } \\
\text { y }\end{array}$ & $\begin{array}{c}\text { Relative } \\
\text { Value }\end{array}$ & $\begin{array}{l}\text { Long- } \\
\text { Only } \\
\text { Absolut } \\
\text { e } \\
\text { Return } \\
\text { Fund }\end{array}$ & $\begin{array}{l}\text { S\&P } \\
500\end{array}$ \\
\hline $\begin{array}{c}\text { Arbitrag } \\
\mathrm{e}\end{array}$ & 1 & & & & & & & & & \\
\hline
\end{tabular}




\begin{tabular}{|c|c|c|c|c|c|c|c|c|c|c|}
\hline $\begin{array}{l}\text { CTA/M } \\
\text { anaged } \\
\text { Futures }\end{array}$ & 0.75 & 1 & & & & & & & & \\
\hline $\begin{array}{l}\text { Distres } \\
\text { sed } \\
\text { Debt }\end{array}$ & 0.45 & 0.25 & 1 & & & & & & & \\
\hline $\begin{array}{l}\text { Fixed } \\
\text { Income }\end{array}$ & 0.81 & 0.69 & 0.20 & 1 & & & & & & \\
\hline $\begin{array}{l}\text { L/S } \\
\text { Equities }\end{array}$ & 0.85 & 0.72 & 0.24 & 0.82 & 1 & & & & & \\
\hline Macro & 0.97 & 0.68 & 0.32 & 0.75 & 0.79 & 1 & & & & \\
\hline $\begin{array}{c}\text { Multi- } \\
\text { Strateg } \\
y\end{array}$ & 0.69 & 0.47 & 0.73 & .40 & 0.49 & 0.58 & 1 & & & \\
\hline $\begin{array}{l}\text { Relativ } \\
\text { e Value }\end{array}$ & 1 & 0.77 & 0.43 & 0.82 & 0.86 & 0.95 & 0.68 & 1 & & \\
\hline $\begin{array}{l}\text { Long- } \\
\text { Only } \\
\text { Absolut } \\
\text { e } \\
\text { Return } \\
\text { Fund }\end{array}$ & 0.86 & 0.80 & 0.27 & 0.00 & 0.84 & 0.79 & 0.52 & 0.88 & 1 & \\
\hline $\begin{array}{l}S \& P \\
500\end{array}$ & 0.01 & 0.01 & 0.01 & 0.00 & 0.04 & 0.01 & 0.00 & 0.00 & 0.03 & 1 \\
\hline
\end{tabular}

\section{ANALYSIS}

The optimal risk portfolio is 5.85\% Arbitrage, $11.63 \%$ CTA/Managed Futures, 10.20\% Distressed Debt, 7.99\% Fixed Income, 10.35\% L/S Equities, 6.56\% Macro, 8.77\% Multi-Strategy, $4.39 \%$ Relative Value, $25.85 \%$ LongOnly Absolute Return Fund, 8.41\% S\&P 500.
The expected monthly return is $0.37 \%$, the monthly standard deviation $1.23 \%$, the annualized Sharpe ratio 1.04 .

We conducted 5-fold cross-validation test and got results as the optimal risky portfolio derived from each test in Table 5, also with the average monthly excess return rates and monthly standard deviation rates of each portfolio in Table 6:

Table 5. shows Optimal Risky Portfolio Derived from Each Test

\begin{tabular}{|c|c|c|c|c|c|c|c|c|c|c|}
\hline & $\begin{array}{c}\text { Arbitrag } \\
\mathrm{e}\end{array}$ & $\begin{array}{l}\text { CTA/M } \\
\text { anaged } \\
\text { Futures }\end{array}$ & $\begin{array}{l}\text { Distress } \\
\text { ed Debt }\end{array}$ & $\begin{array}{l}\text { Fixed } \\
\text { Income }\end{array}$ & $\begin{array}{c}\text { L/S } \\
\text { Equities }\end{array}$ & Macro & $\begin{array}{c}\text { Multi- } \\
\text { Strateg } \\
y\end{array}$ & $\begin{array}{c}\text { Relative } \\
\text { Value }\end{array}$ & $\begin{array}{l}\text { Long- } \\
\text { Only } \\
\text { Absolut } \\
\text { e } \\
\text { Return } \\
\text { Fund }\end{array}$ & $\begin{array}{l}S \& P \\
500\end{array}$ \\
\hline $\begin{array}{c}01 / 01- \\
04 / 04 \\
\text { as Test } \\
\text { Set }\end{array}$ & $4.36 \%$ & $8.42 \%$ & $8.96 \%$ & $9.11 \%$ & $9.40 \%$ & $7.07 \%$ & $5.28 \%$ & $-0.69 \%$ & $22.73 \%$ & $25.36 \%$ \\
\hline
\end{tabular}




\begin{tabular}{|c|c|c|c|c|c|c|c|c|c|c|}
\hline $\begin{array}{c}05 / 04- \\
07 / 08 \\
\text { as Test } \\
\text { Set }\end{array}$ & $12.61 \%$ & $10.00 \%$ & $10.96 \%$ & $11.17 \%$ & $5.22 \%$ & $6.72 \%$ & $6.36 \%$ & $3.81 \%$ & $16.63 \%$ & $16.51 \%$ \\
\hline $\begin{array}{c}08 / 08- \\
10 / 12 \\
\text { as Test } \\
\text { Set }\end{array}$ & $12.44 \%$ & $11.34 \%$ & $11.98 \%$ & $6.47 \%$ & $7.17 \%$ & $8.96 \%$ & $8.32 \%$ & $6.66 \%$ & $14.81 \%$ & $11.86 \%$ \\
\hline $\begin{array}{c}11 / 12- \\
01 / 17 \\
\text { as Test } \\
\text { Set }\end{array}$ & $9.44 \%$ & $17.85 \%$ & $8.55 \%$ & $9.17 \%$ & $4.38 \%$ & $12.72 \%$ & $7.27 \%$ & $1.13 \%$ & $21.84 \%$ & $7.64 \%$ \\
\hline $\begin{array}{c}02 / 17- \\
04 / 21 \\
\text { as Test } \\
\text { Set }\end{array}$ & $4.43 \%$ & $15.56 \%$ & $17.97 \%$ & $10.86 \%$ & $2.56 \%$ & $12.93 \%$ & $6.93 \%$ & $2.16 \%$ & $20.26 \%$ & $6.33 \%$ \\
\hline
\end{tabular}

Table 6. shows Average Monthly Excess Return Rate and Monthly Standard Deviation of Each Optimal Risky Portfolio

\begin{tabular}{|c|c|c|c|c|}
\hline & $\begin{array}{c}\text { Average Monthly } \\
\text { Excess Return Rate } \\
\text { of Training Set }\end{array}$ & $\begin{array}{c}\text { Monthly Standard } \\
\text { Deviation of Training } \\
\text { Set }\end{array}$ & $\begin{array}{c}\text { Average Monthly } \\
\text { Excess Return Rate } \\
\text { of Test Set }\end{array}$ & $\begin{array}{c}\text { Monthly Standard } \\
\text { Deviation of Test Set }\end{array}$ \\
\hline $\begin{array}{c}01 / 01-04 / 04 \text { as } \\
\text { Test Set }\end{array}$ & $0.43 \%$ & $1.89 \%$ & $0.21 \%$ & $1.76 \%$ \\
\hline $\begin{array}{c}05 / 04-07 / 08 \text { as } \\
\text { Test Set }\end{array}$ & $0.35 \%$ & $1.28 \%$ & $0.29 \%$ & $2.18 \%$ \\
\hline $\begin{array}{c}08 / 08-10 / 12 \text { as } \\
\text { Test Set }\end{array}$ & $0.38 \%$ & $1.23 \%$ & $0.07 \%$ & $0.98 \%$ \\
\hline $\begin{array}{c}11 / 12-01 / 17 \text { as } \\
\text { Test Set }\end{array}$ & $0.34 \%$ & $1.67 \%$ & $0.39 \%$ & $1.30 \%$ \\
\hline $\begin{array}{c}02 / 17-04 / 21 \text { as } \\
\text { Test Set }\end{array}$ & $0.33 \%$ & $1.48 \%$ & $0.19 \%$ & \\
\hline
\end{tabular}

We can conclude that our method to build the optimal risky portfolio generally worked well by comparing the average monthly excess return rates and the monthly standard deviation of training sets to those of test sets.

\section{CONCLUSION}

The goal of this paper is to identify some welldeveloped hedge fund portfolios aiming at managing risks. It was written to inform its readers of our thoughts on risk management and offer some strategies through careful analysis. By utilizing hedge fund's characteristics and analyzing their past statistics, we identified the optimal risk portfolios which contained a total of nine strategies of hedge funds and S\&P 500 that will most likely obtain an appropriate level of risk management for investors. Our suggestion is to put $91.59 \%$ of your total money in hedge fund, which is, 5.85\% in Arbitrage, $11.63 \%$ in CTA/Managed Futures, $10.20 \%$ in Distressed Debt, $7.99 \%$ in Fixed Income, 10.35\% in L/S Equities, 6.56\% in Macro, $8.77 \%$ in Multi-Strategy, $4.39 \%$ in Relative Value, $25.85 \%$ in Long-Only Absolute Return Fund, and $8.41 \%$ in S\&P 500. By such allocation method, the annualized Sharpe ratio of our optimal risky portfolio is 1.04, compared to the average level of different strategies of hedge funds, 0.63 , is $64.73 \%$ higher; compared to Arbitrage, the highest single strategy of hedge fund, 0.74 , is $39.30 \%$ higher; compared to S\&P 500, 0.32, is $227.37 \%$ higher, which is even more drastic. Hedge funds are efficient tools for investors as they can help them achieve 
high risk management while still being capable of claiming higher returns by posing higher risks. Since open-ended, however, hedge fund's potential far exceeds the presented portfolios, ultimately providing investors opportunities to elaborate their existing strategy and expand their capitals [1-6]. Our portfolio provided a method to generate a relatively higher return and lower risk, and we proved that such method tends to be of low bias by 5 -fold cross-validation test.

\section{REFERENCES}

[1] Cowell, Frances. Hedge Funds: A Risk Manager's Viewpoint, Aug. 2010, www.eurekahedge.com/Research/News/401/Hedge -Funds-A-Risk-Managers-Viewpoint.

[2] Goltz, Felix. "Hedge Funds as Risk Reducers." IPE, IPE, 4 Feb. 2021, www.ipe.com/hedge-funds-asrisk-reducers/19973.article.

[3] Bruder, Benjamin, and Serge Darolles. "(PDF) Portfolio Allocation of Hedge Funds." ResearchGate, Jan. 2010, www.researchgate.net/publication/228205815_Port folio_Allocation_of_Hedge_Funds.

[4] Building Efficient Hedge Fund Portfolios - Fiera Capital. Fiera Capital Inc., Aug. 2017, us.fieracapital.com/wpcontent/uploads/2017/10/building-efficient-hedgefund-portfolios-10.9.17.pdf.

[5] Cassar, Gavin, and Joseph Gerakos. How Do Hedge Funds Manage Portfolio Risk? Dec. 2010, www.top1000funds.com/wpcontent/uploads/2011/01/HowDoHedgeFundsMana gePortfolioRisk.pdf.

[6] Iordanidis, Kostas. (PDF) Hedge Fund Investment Philosophy: A Methodology for ... Mar. 2017, www.researchgate.net/publication/319666463_Hed ge_Fund_Investment_Philosophy_A_methodology _for_selecting_hedge_fund_managers_and_constru cting_hedge_fund_portfolios.

[7] Browne, Michael W. “(PDF) Cross-Validation Methods." Journal of Mathematical Psychology, Volume 44, Issue 1, March 2000, Pages 108-132, https://www.sciencedirect.com/science/article/abs/ pii/S0022249699912798 